\title{
Algebraic Turbulence-Chemistry Interaction Model
}

\author{
A. T. Norris * \\ NASA Langley Research Center, Hampton, Virginia, 23681-2199, U.S.A
}

\begin{abstract}
The results of a series of Perfectly Stirred Reactor (PSR) and Partially Stirred Reactor (PaSR) simulations are compared to each other over a wide range of operating conditions. It is found that the PaSR results can be simulated by a PSR solution with just an adjusted chemical reaction rate. A simple expression has been developed that gives the required change in reaction rate for a PSR solution to simulate the PaSR results. This expression is the basis of a simple turbulence-chemistry interaction model. The interaction model that has been developed is intended for use with simple one-step global reaction mechanisms and for steady-state flow simulations. Due to the simplicity of the model there is very little additional computational cost in adding it to existing CFD codes.
\end{abstract}

\section{Introduction}

Due to the non-linear nature of the governing equations for turbulent reacting flow, it is well known that the expression for the mean species source terms due to chemical reaction are not being calculated exactly. Specifically, using the mean species concentrations and mean temperature to calculate the mean species source term is not accurate. Indeed it has been shown that the differences in the mean reaction rate associated with this approximation can be several orders of magnitude different from the exact solution. ${ }^{1}$

To this end, several models and numerical approaches have been developed in order to resolve this issue. Models include the Magnussen model, ${ }^{2}$ the Direct Quadrature Moment Method (DQMOM) ${ }^{3}$ the Assumed PDF ${ }^{4}$ the Lagrangian $\mathrm{PDF}^{5}$ and the Linear Eddy Model. ${ }^{6}$ All these models attempt to model the effect that turbulence has on chemical reaction with varying degrees of fidelity. Unfortunately, as the modeling becomes more sophisticated, there is a corresponding increase in the computational cost. Of all the models listed above, the one with the least computational expense is the Magnussen model, and as a result, it is very popular. However the simplicity of the model does result in it lacking several desirable features.

Let us consider a chemical reaction defined by the mass fraction of Fuel $Y_{f}$, Oxidizer $Y_{o}$ and Products $Y_{p}$. The fuel reaction rate for the Magnussen model is given by:

$$
\frac{d Y_{f}}{d t}=\operatorname{Min}\left\{\begin{array}{l}
A Y_{f} / t_{t} \\
A Y_{o} / t_{t} r_{s} \\
B Y_{p} / t_{t}\left(1+r_{s}\right)
\end{array}\right.
$$

where $A$ and $B$ are constants, $r_{s}$ is the stoichiometric ratio of fuel to oxidizer and $t_{t}$ is the turbulent time scale. It is apparent that in this form, the effect of the turbulence on chemistry is modeled by simple making the reaction rate inversely proportional to the turbulent time scale and thus as the turbulent time scale changes, so does the reaction rate.

\footnotetext{
*Aerospace Engineer, Hypersonic Airbreathing Propulsion Branch, Senior Member AIAA.
} 
However real chemical reactions have an upper limit to the reaction rate, which is a not accounted for in this model. A simple modification to fix this is to use a global mechanism to limit the reaction rate, such as those developed by Westbrook and Dryer. ${ }^{7}$ Thus the reaction rate would be give by

$$
\frac{d Y_{f}}{d t}=\operatorname{Min}\left\{\begin{array}{l}
d Y_{f} / d t \text { (Magnussen) } \\
C Y_{f}^{a} Y_{o}^{b} \exp (-E / R T)
\end{array}\right.
$$

where $C, a, b, E$ and $R$ are the global rate constants and $T$ is the temperature. However this simple truncation is a somewhat ad-hoc approach and a more rigorous approach would be desirable.

The goal of this paper is to investigate whether such a model can be developed that has the numerical simplicity of the Magnussen model yet have some of the desirable physical effects of the more complex methods. The objective is not to develop a model that works for complex finite-rate chemistry problems, but rather one that gives a good approximation of reacting flow for a minimum level of computational effort. A Magnussen-like reaction rate model, or a simple one-step global mechanism would be examples of the target chemical models.

As an approach to obtaining this improved simple model, consider a single computational cell in a finitevolume CFD solution with chemical reaction (figure 1) where $m_{j}$ is the mass of species $j$ contained in the

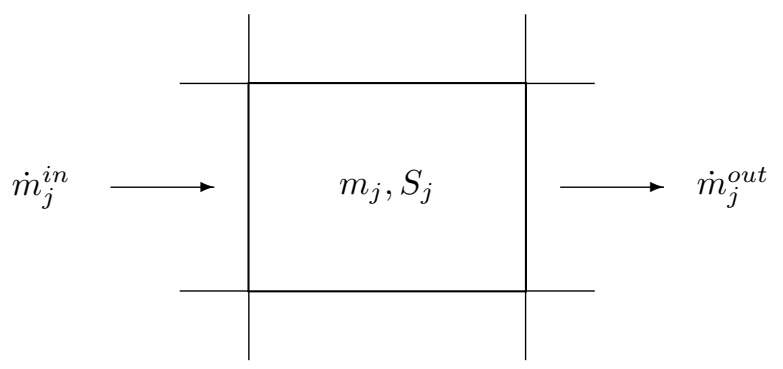

Figure 1. Schematic of single computational cell in a finite volume CFD solution.

cell and $S_{j}$ is the rate of creation of species $j$ due to chemical reaction. Also shown is the mass flow rate of species $j$ entering the cell, $\dot{m}_{j}^{i n}$ and the mass flow rate leaving the cell, $\dot{m}_{j}^{\text {out }}$. The change in the mass contained in the cell as a function of time is given by

$$
\frac{d m_{j}}{d t}=S_{j}+\dot{m}_{j}^{i n}-\dot{m}_{j}^{\text {out }} .
$$

For a steady state situation, which would correspond to a steady state CFD solution, the left hand side of equation 3 is zero, and it is apparent that there is a strong resemblance between this single cell and a Perfectly Stirred Reactor, (PSR). The mass contained in the cell, divided by the inflow mass flux gives a residence time, $t_{r}$ and the composition of the cell can be plotted simply as a function of residence time.

The results of a typical PSR calculation are shown in figure 2. As the mass flux into the reactor increases, the residence time decreases and the mass fraction of products of combustion start to drop off. When the inflow becomes sufficiently large, the reaction is unable to be sustained, and the reactor is said to have blown out. For a given volume then, the reactor is parameterized by just the residence time and reaction rate. 


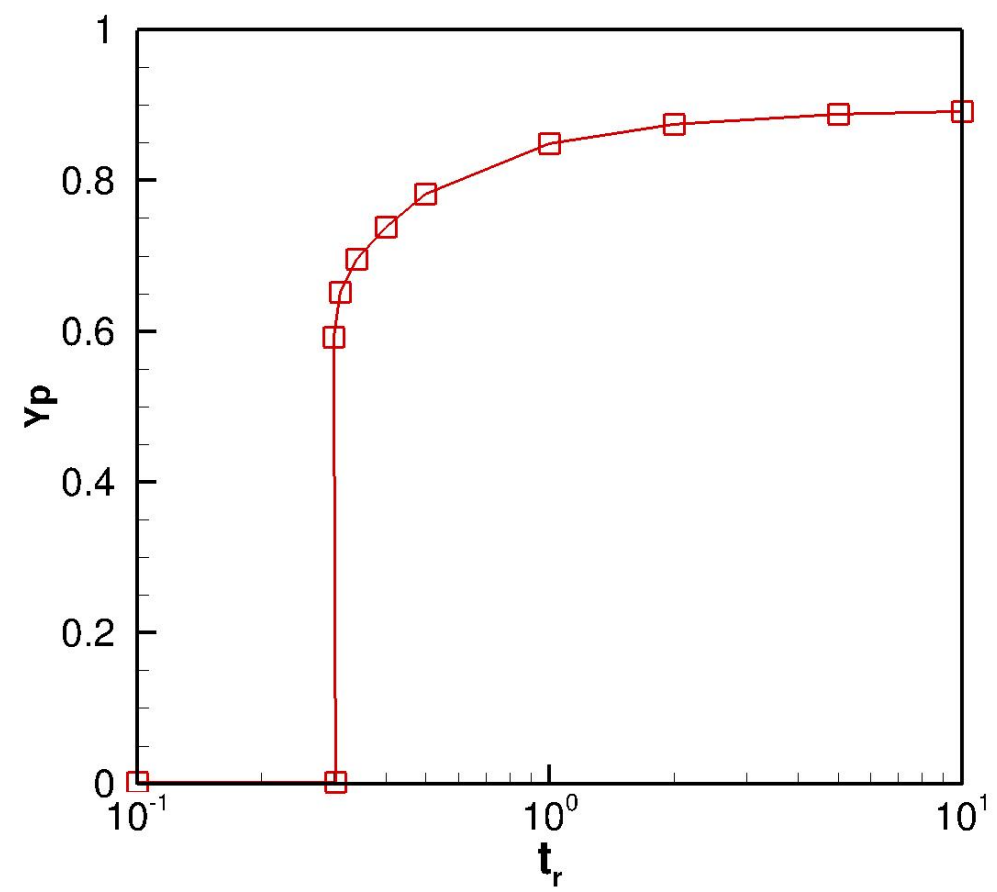

Figure 2. Results of a typical PSR simulation showing the progress variable $Y_{p}$ plotted as a function of the reactor residence time $t_{r}$.

Likewise, consider a single cell in an Eulerian Probability Density Function (PDF) simulation shown in figure 3 .

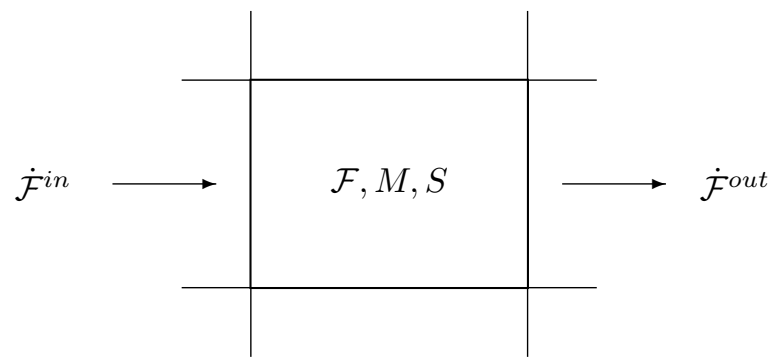

Figure 3. Schematic of a single computational cell in an Eulerian Probability Density Function simulation.

The composition in the cell is represented by a probability distribution of different values of scalar mass fractions, $\mathcal{F}_{j}$, which evolves due to molecular mixing, $M$, chemical reaction, $S$ and the inflow and outflow of distributions of species $j$, given by $\dot{\mathcal{F}}^{\text {in }}$ and $\dot{\mathcal{F}}^{\text {out }}$ respectively. The evolution of the distribution of all the scalar quantities in the cell, $\mathcal{F}$ as a function of time is given by

$$
\frac{d \mathcal{F}(\psi, t)}{d t}=\sum_{j=1}^{N_{s}} \frac{d}{d \psi_{j}}\left[M_{j}(\psi, t)+S_{j}(\psi, t)\right] \mathcal{F}(\psi, t)+\dot{\mathcal{F}}^{\text {in }}(\psi)-\dot{\mathcal{F}}^{\text {out }}(\psi)
$$

where the $\psi_{j}$ represents the scalar space of species $j$ and the number of scalars is given by $N_{s}$. In a similar way as with the PSR, if the change of the distribution of scalars, $\mathcal{F}$ with time is zero, corresponding to a steady state solution, the computational cell can be considered to behave the same as a Partially Stirred 
Reactor, (PaSR). Like the PSR, the PaSR is parameterized by a residence time scale and a reaction time scale, but also has a mixing time scale.

While equation 3 and equation 4 look very different they are in fact very similar. If the distribution $\mathcal{F}$ is set to a dirac delta function, and molecular mixing is not considered, equation 3 is recovered for each species $j$. That is, a PSR can be thought of as a PaSR but without the molecular mixing term. Thus by comparing the results of a PSR simulation with the mean PaSR quantities, the effect of the interaction between chemistry and turbulence can be evaluated in a consistent manner, and the possibility of a simple relationship between the results may be possible.

\section{Discussion}

To investigate the relationship between a PSR and a PaSR, the first thing to do is to develop a suitable set of reactor models, and then test them over a range of conditions that might be encountered in a reacting flow. In this section the model used for the reactor and the chemistry model are described. The results of a comprehensive series of simulations that compare the PSR and PaSR results are also described.

\section{A. Reactor Model}

In order to compare the results of a PSR and PaSR model, it is important to eliminate any discrepancies that may be caused by different numerical techniques. To this end, both the PaSR and the PSR were modeled using a particle-based stochastic model. The stochastic method uses an ensemble of particles $N_{p}$, to represent the scalar composition within a reactor. During a time step, a certain number of particles enter the reactor, $N^{i n}$, and an equal number leave. The particles are then mixed via a molecular mixing model and the resulting composition is then reacted and the process repeats.

For this study, three molecular mixing models were used: A Perfect Mixing Model (PMM), the Interaction and Exchange with the Mean (IEM) model $^{8}$ and the Modified Curl Model. ${ }^{9}$

The PMM takes all the particles in the reactor, and sets their scalar values to the corresponding ensemble mean value. For example, for a given time step $\delta t$, the value of particle $\phi^{p}$ changes as follows:

$$
\phi^{p}(t+\delta t)=\bar{\phi}(t)
$$

where $\bar{\phi}$ is the ensemble mean value. The perfect mixing model, as the name suggests is used to model the PSR.

For the PaSR, two models are used. The simpler model is the IEM scheme, which assumes the scalar value of the particles will approach the scalar ensemble mean value at an exponential rate governed by the turbulent time scale, $t_{t}$. The change in a particle $\phi^{p}$ for a time step of $\delta t$ is given by:

$$
\phi^{p}(t+\delta t)=\bar{\phi}+\left(\phi^{p}(t)-\bar{\phi}\right) \exp \left(-\frac{1}{2} C_{\phi} \frac{\delta t}{t_{t}}\right)
$$

where $C_{\phi}$ is the mixing constant, with a value commonly set to unity.

The other PaSR molecular mixing model used is the modified Curl model. This is a particle interaction model based on the original Curl model. ${ }^{10}$ In this model, a pair of particles, $\phi^{p}$ and $\phi^{q}$ are randomly selected from the ensemble, and their compositions are changed to

$$
\begin{aligned}
& \phi^{p}(t+\delta t)=\phi^{p}(t)+\frac{1}{2} r\left(\phi^{q}(t)-\phi^{p}(t)\right) \\
& \phi^{q}(t+\delta t)=\phi^{q}(t)+\frac{1}{2} r\left(\phi^{p}(t)-\phi^{q}(t)\right)
\end{aligned}
$$

where $r$ is a random number with a uniform distribution in the interval $(0,1)$. In this case however the time step $\delta t$ is a function of the turbulent time scale and number of particles in the ensemble, and is given by:

$$
\delta t=\frac{t_{t}}{3 N_{p}}
$$

If more than one pair of particles are selected at once, the $\delta t$ can be scaled appropriately. 
In the operation of the PSR and the PaSR model, the particles are initialized with fully mixed and reacted particles. The particles that are to be input into the reactor each turn are selected with either a mean value or from a specified scalar distribution.

The overall time step used is based on the number of particles that are selected to be input, however the mixing and reaction steps are often run at a smaller time step for several sub iterations to ensure certain numerical constraints, such as the time step required for the Curl mixing model. For each run, the code keeps iterating until the mean scalar quantities in the reactor have converged to a statistically constant value. The mean scalar quantities are evaluated using a time-averaging technique to reduce the amount of scatter in the results.

During the simulations reported in this paper, the results for the modified Curl model are the ones predominately reported. However the results for the IEM model have also been performed, and show very similar results to those of the modified Curl model.

\section{B. Reaction Model}

The desired reaction model for this study would be a global one-step reaction, where the species are represented by a minimum number of scalar variables. For example the Magnussen model can be represented by the mass fraction of fuel, oxidizer and products. This can also be reformulated as simply a mixture fraction $\xi$ and a progress variable $Y_{p}$.

For the purposes of this study, a simple polynomial representation for chemical reaction was developed based on $\xi$ and $Y_{p}$. The motivation for this is to have a mechanism that has the same form as a typical one-step global mechanism, yet has an algebraic integral solution that will allow a very efficient solution for the PSR and PaSR code. This will afford the opportunity to perform many simulations with a minimum of computational effort. The polynomial rate equation chosen is given as

$$
\frac{d Y_{p}}{d t}=A Y_{p}^{3}\left(\hat{Y}_{p}(\xi)-Y_{p}\right)
$$

where $A$ is the reaction rate constant, and $\hat{Y}_{p}(\xi)$ is the maximum value of the progress variable as a function of the mixture fraction, $\xi$. In figure 4 the reaction rate of this chemistry model is compared to scaled versions of the Magnussen model and a typical one-step global reaction.

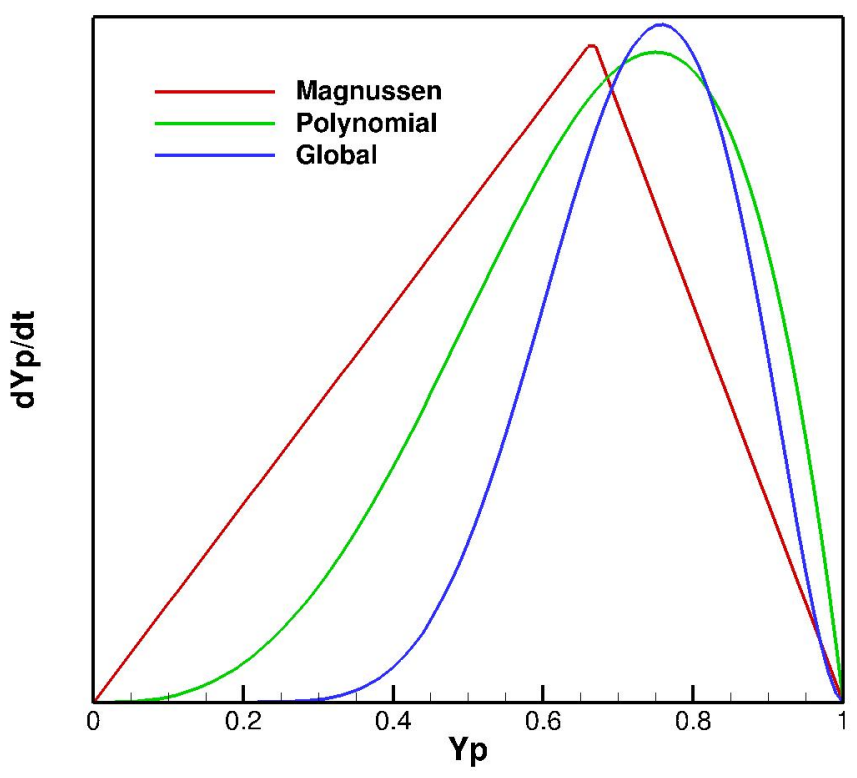

Figure 4. The progress variable reaction rate $d Y_{p} / d t$ plotted as a function of the progress variable $Y_{p}$ for three different reaction mechanisms. 
The rate expression in equation 10 can be integrated to obtain an expression for the progress variable as a function of time $t$,

$$
t=\frac{1}{A \hat{Y}_{p}^{2}(\xi)}\left[\frac{1}{\hat{Y}_{p}(\xi)} \log \left[\frac{Y_{p}}{\hat{Y}_{p}(\xi)-Y_{p}}\right]-\frac{2 Y_{p}+\hat{Y}_{p}(\xi)}{2 Y_{p}^{2}}\right],
$$

which is solved via a bisection method. For this study, the value of $A=20$ was chosen to give a residence time for the reactors of between 0.1 and 10 .

The inverse of the maximum reaction rate for a given value of mixture fraction can be used as a characteristic time for the reaction. Thus the reaction time scale, $t_{x}$ for equation 10 is given by,

$$
t_{x}=\frac{16}{3 A \hat{Y}_{p}(\xi)^{4}}
$$

\section{Numerical Validity}

To establish the numerical validity of the PSR and PaSR solutions it is important to investigate the numerical aspects of the solution process. To this end the numerical parameters controlling the solution were varied to obtain a range of operability for the reaction code.

The first parameter varied was the Particle Replacement Ratio (PRR) number, defined in this effort as the number of particles entering the reactor per time step relative to the number of particles contained by the reactor. Operating parameters chosen for this were $N_{p}=1000$ particles and a turbulence time scale of $t_{t}=0.2$. The results for the modified Curl model simulation are shown in figure 5 , where it is apparent that the effect of the PRR number on the solution is largest at a residence time of $t_{r}=1$. Similar results were also obtained for the PSR and RTM simulations.

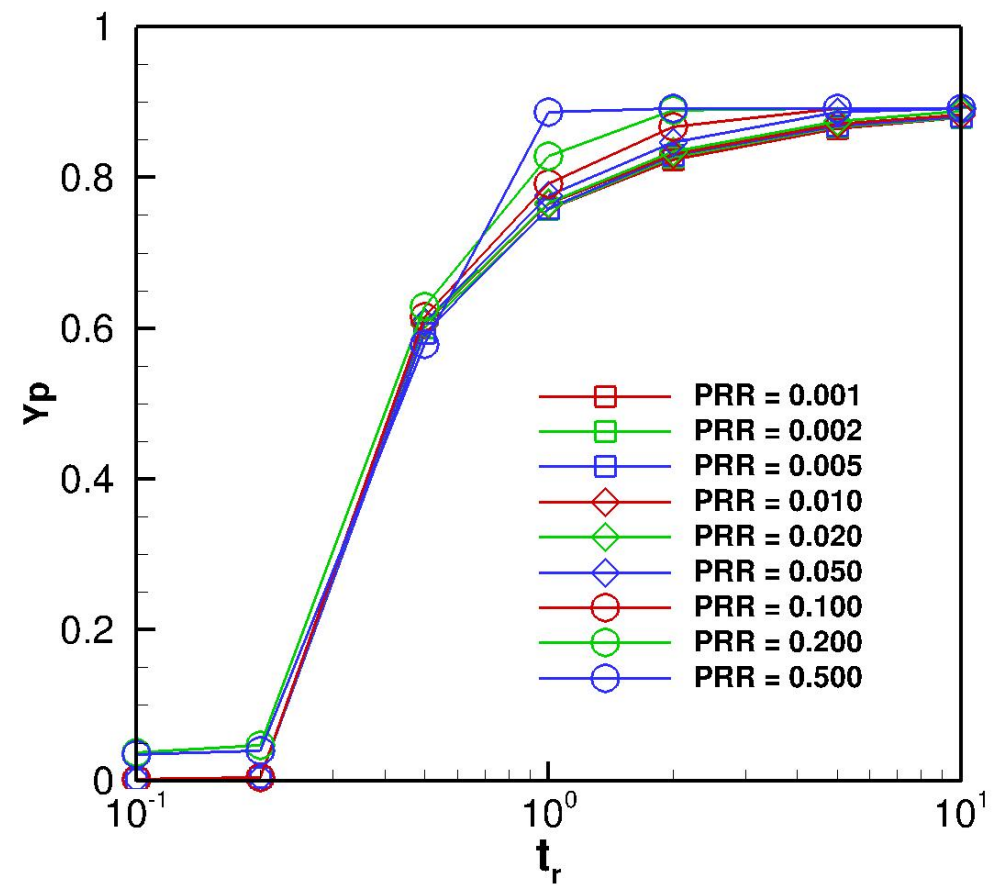

Figure 5. The progress variable $Y_{p}$ plotted as a function of the reactor residence time $t_{r}$ showing the effect of varying the particle replacement ratio (PRR). Curl mixing model used.

Plotting the progress variable against the PRR number for all three mixing models, figure 6 , shows that the solutions for all models are numerically insensitive for a PRR of less that 0.02 , so this value will be used in all further simulations. It is interesting to note that the Curl and the RTM model do not converge to 


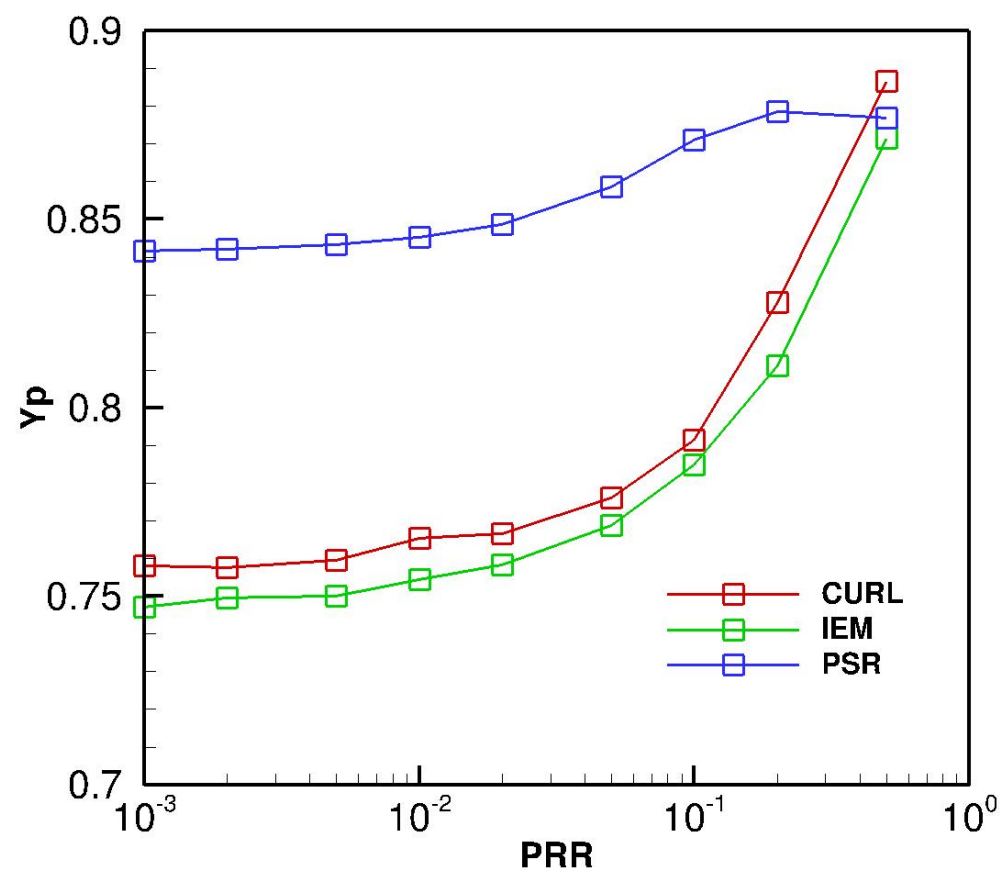

Figure 6. The progress variable $Y_{p}$ plotted as a function of the Particle Replacement Ratio (PRR). Results shown for the PaSR reactor simulations using the modified Curl model and the IEM mixing model and compared to the PSR model.

the same value despite being expected to provide the same level of mixing. The PSR simulation though was expected to converge to a different value as it assumes total mixing.

The number of particles required for an accurate solution was also investigated. Using the same initial conditions as above, except with the PRR number set to 0.02 , and varying the total number of particles gives a result for the Curl model shown in figure 7. It is apparent from this plot that using $N=100$ particles gives almost identical results to cases using $N=1000$ particles. Thus solving cases with $N=100$ particles was deemed adequate for all further simulations. Similar results were obtained for the RTM mixing model and the PSR.

For the Curl model the number of particles from the ensemble that are selected for mixing is another numerical parameter. Tests were performed and the results were found to be insensitive to the number of mixing particles selected. For all further simulations, a maximum of $20 \%$ of the ensemble particles were selected for mixing each time step. If more particles were required, the mixing and reaction were looped over multiple times to ensure the $20 \%$ limit was kept to.

\section{Physical Variations}

The first physical parameter varied was the reaction time scale and the PSR results are shown in figure 8 . As expected, the effect of changing the reaction rate is to simply shift the curve along the residence time axis. Doubling the reaction time scale doubles the residence time that the reactor requires to maintain a given level of combustion. A similar result was obtained for the PaSR simulation, with the added requirement that the ratio of the turbulent and reactive time scales was kept constant.

The next test was to vary the turbulence time and the PaSR results for the Curl model are shown in figure 9. It can be seen in this plot that as the turbulence time scale decreases the PaSR result approaches the PSR result. Thus there is an limit to which turbulence will enhance the chemical reaction in a PaSR, which is an effect that the basic Magnussen model does not account for. In comparing the curves produced by different turbulence levels in figure 9 it can be seen that the shapes are fairly similar to that of the PSR result, just moved to the right as the turbulence time scale increases. Coupling this with the result observed 


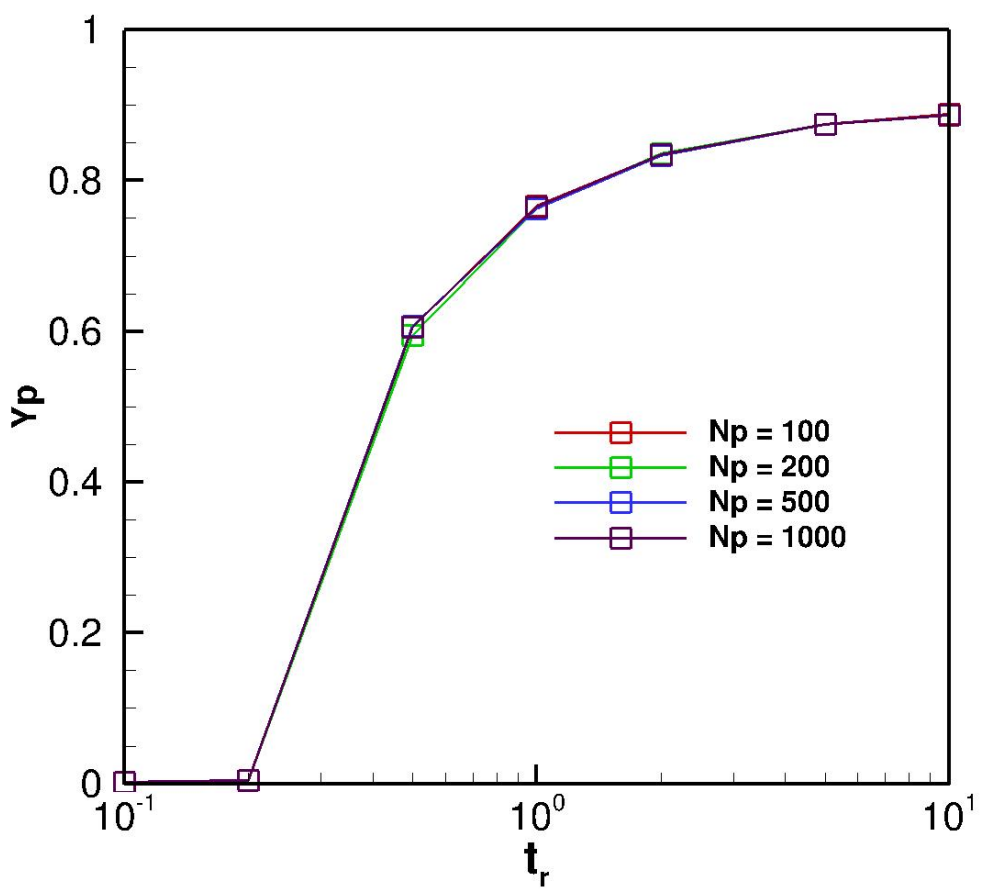

Figure 7. Effect of changing the number of particles $N_{p}$ used to represent the PaSR.

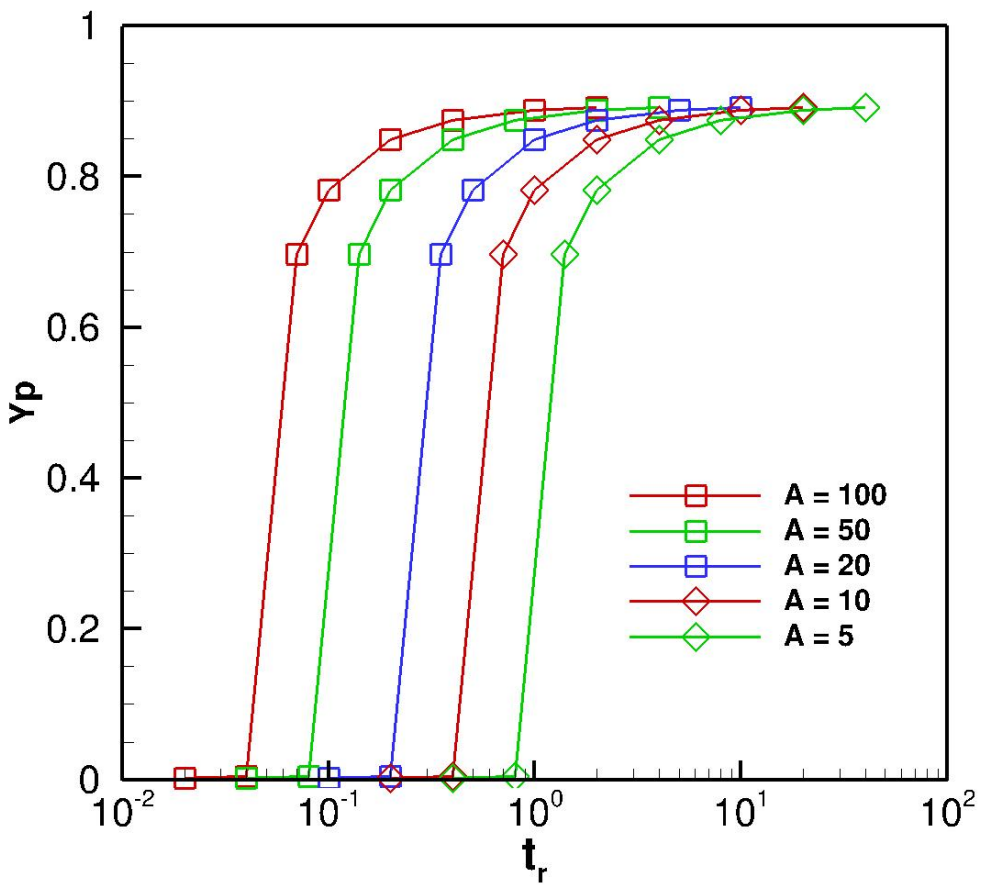

Figure 8. Results of PSR simulations with different values of reaction rate. 


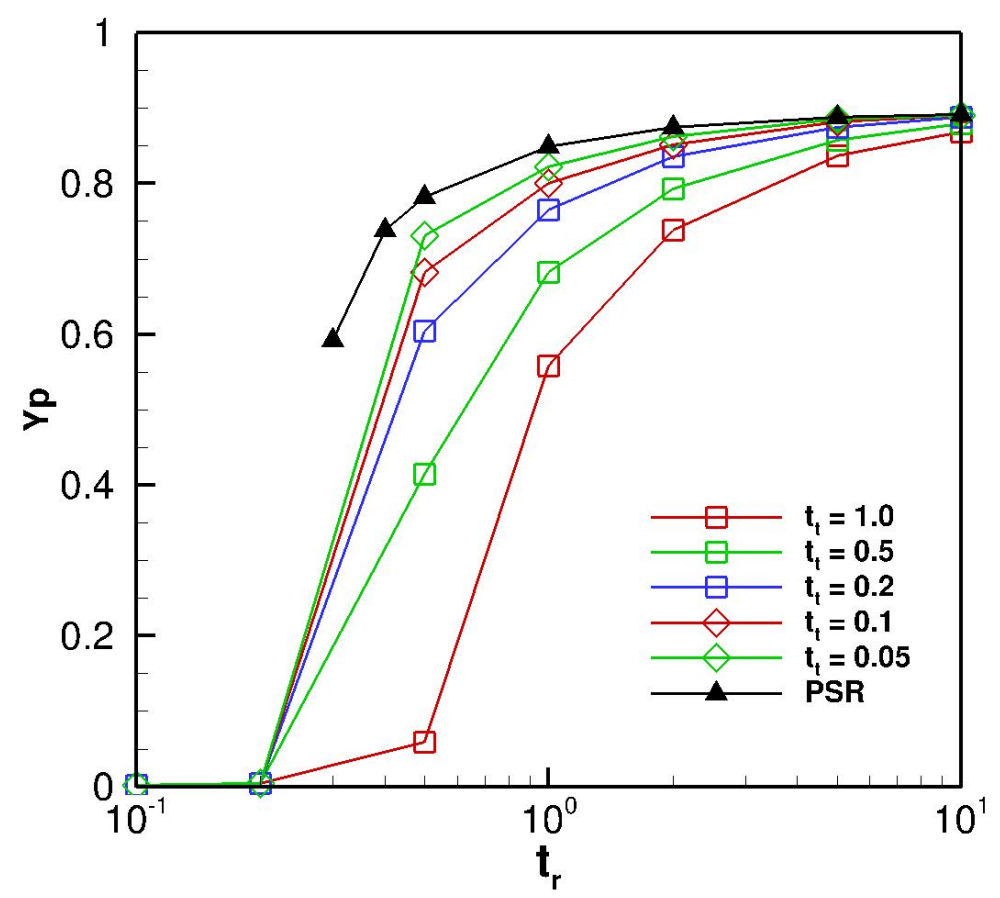

Figure 9. Results of Curl model PaSR simulations for different turbulence time scales. PSR result shown for reference.

in figure 8 where the PSR curve moves proportional to the reaction rate, a scheme based on changing the reaction rate of a PSR to simulate the effects of turbulence in a PaSR should be possible. The expression for this model is given by:

$$
A=\frac{A_{o}}{\left(1+D_{t} t_{t} / t_{x}\right)}
$$

where $A_{o}$ is the original reaction rate constant, $A$ is the new modeled value and $D_{t}$ is a constant which has a fitted value of $D_{t}=2.1$ for the kinetic mechanism used in this study.

The reactor results produced by this expression are shown plotted against the PaSR results for a range of turbulence time scales in figure 10. It can be seen that this model does provide a reasonably good approximation of the PaSR results. It certainly matches the trend of increased turbulence driving the PaSR solution to approach the PSR result. It should be noted that the agreement for lower values of $Y_{p}$ is not so good, but as simple chemical mechanisms have considerable uncertainty in this region it is not considered a significant flaw.

Thus for a given set of conditions for a PaSR, the same result can be obtained by a PSR by simply manipulating the reaction time scale. This result is the basis of the proposed turbulence-chemistry interaction model described in this paper. However in a combustion simulation, the composition entering a computational cell will not be a fixed value, and so it is important to investigate the effects of varying the inflow distributions of scalar quantities.

To this end, a simple test was performed that varied the mean progress variable inflow value, $\bar{Y}_{p}^{i n}$. Sample PaSR results of $\bar{Y}_{p}^{i n}=0.2$ and $\bar{Y}_{p}^{i n}=0.6$ are shown in figure 11 . What is seen here is that as $\bar{Y}_{p}^{i n}$ approaches the fully burnt value, the effect of turbulent mixing becomes smaller. Thus the model can be modified by the mean inflow $\bar{Y}_{p}^{i n}$ as shown:

$$
A=\frac{A_{o}}{\left(1+D_{t}\left(1-\bar{Y}_{p}^{i n} / \hat{Y}_{p}(\xi)\right)^{d_{t}} t_{t} / t_{x}\right)}
$$

where $d_{t}$ is a constant that is found to fit the data with $d_{t}=3.6$. Applying this correction gives results shown in figure 12 where it can be seen that a reasonably good agreement has been obtained between the PaSR results and the model predictions. 


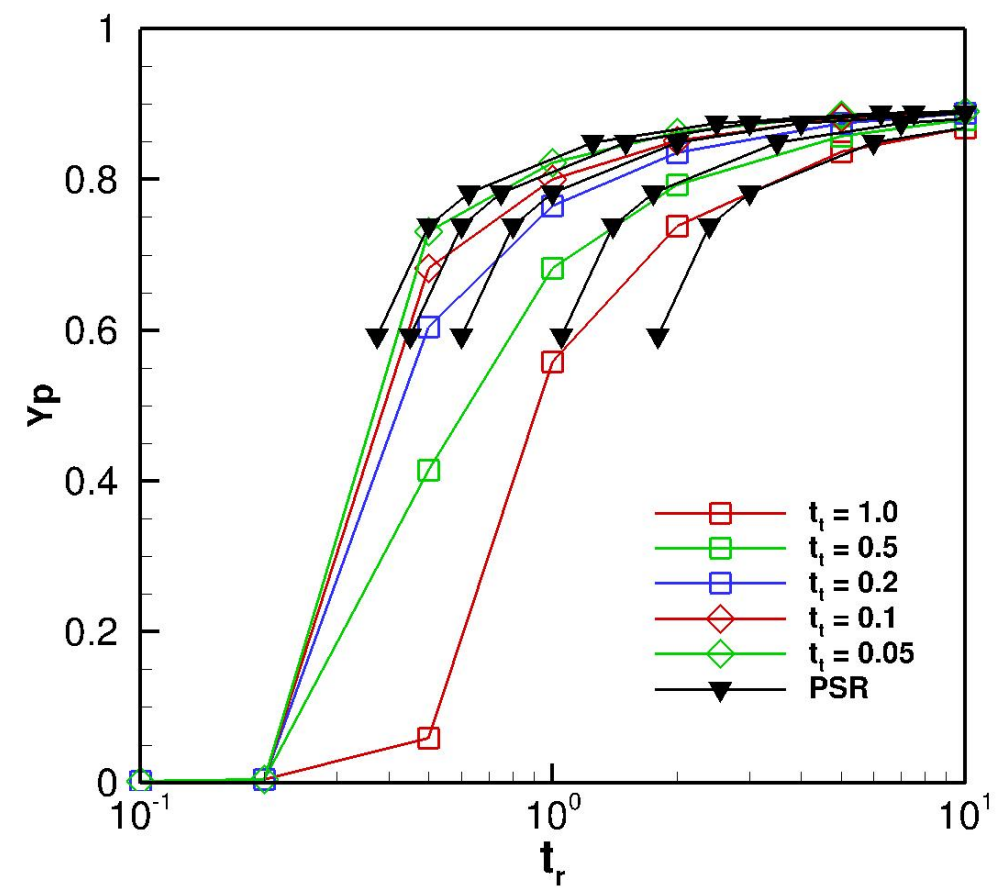

Figure 10. Curl model PaSR simulation results for different turbulent time scales with modified PSR results overlayed.

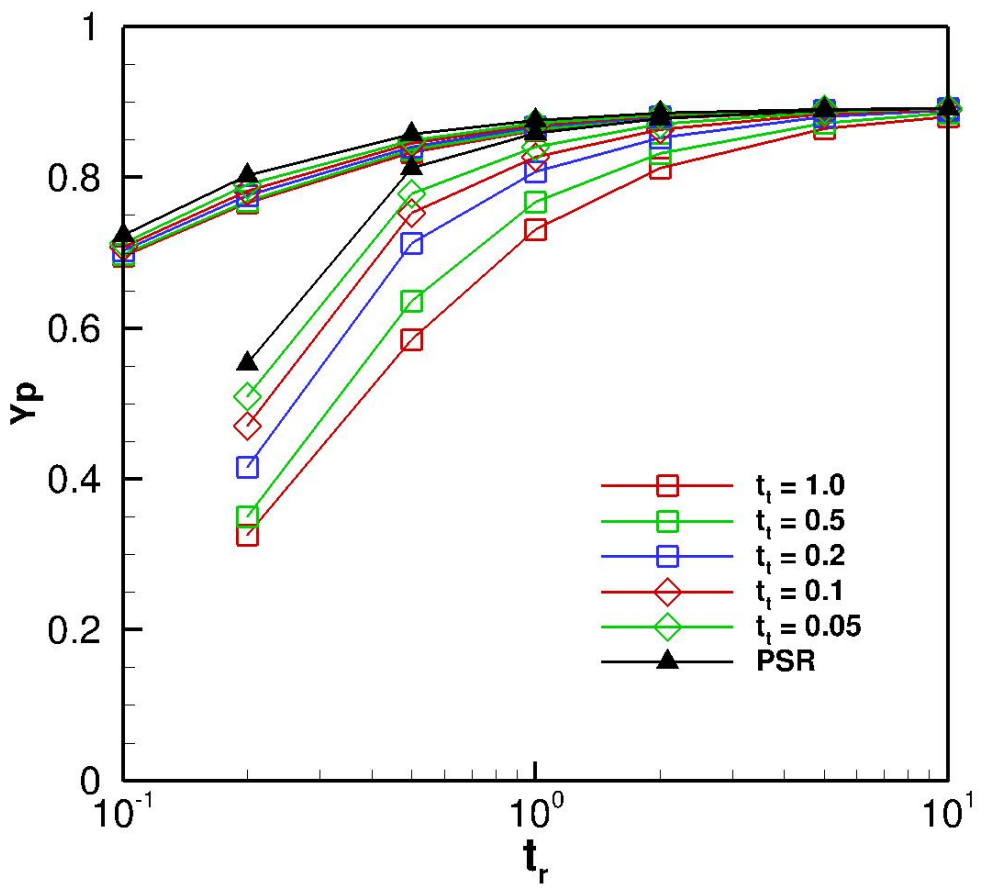

Figure 11. Effect of varying mean inflow progress variable value on Curl model PaSR. Lower lines represent a $\bar{Y}_{p}=0.2$ and upper lines are $\bar{Y}_{p}=0.6$. 


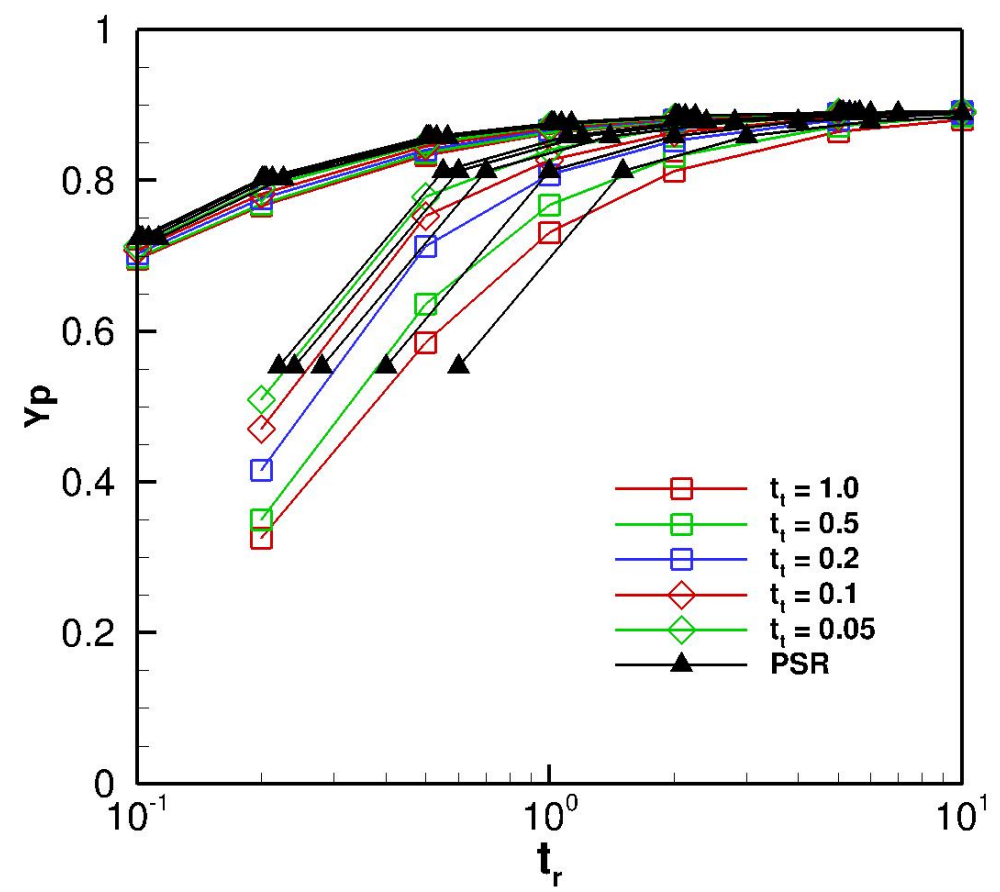

Figure 12. Results of PSR model with variable inflow composition shown overlayed on Curl PaSR model. Lower lines represent a $\bar{Y}_{p}=0.2$ and upper lines are $\bar{Y}_{p}=0.6$.

In all the tests so far, the inflow scalar quantities values have just been set to a mean value. However in full PDF simulations, the value of the inflow scalar quantities do not just have a single value, but are represented by a distribution of values. Because the main goal of this effort was to develop a simple, computational efficient model, any effect of a distribution of inflow scalars would ideally be small so they can be ignored.

To test this, a $\beta$ distribution of $Y_{p}^{i n}$ was used to generate the inlet quantities. With a range of mean progress variable values and standard deviations, $\sigma_{Y p}=0.02,0.1,0.2$ and 0.3 to ensure a wide range of possible input scenarios. A sample of the PDFs of the distributions are shown plotted in figure 13 for a mean normalized progress variable of 0.2. A sample of the PaSR results are shown in figure 14 where the inflow conditions are $\bar{Y}_{p}^{i n}=0.2$ for four different standard deviations and shown for two different turbulent time scales. It can be seen that even for large variations in the distribution, the results are relatively insensitive to the precise distribution and so this effect can be ignored.

So far in this study, the effects of varying the mixture fraction have not been addressed, as this is a conserved scalar. However there can be an effect on the mean progress variable when there is a distribution of $\xi$. To test this, a relationship between the maximum progress variable $Y_{p m}$ and the mixture fraction $\xi$ was chosen and is shown plotted in figure 15. By testing at the stoichiometric value $\xi_{s}=0.2$ it is expected that the PaSR results will be the most effected, as all non-stoichiometric values of mixture fraction will exhibit lower reaction rates than the mean value of $\xi$ and so drag the results lower. A beta distribution of $\xi$ values was chosen to represent possible distributions, with a mean $\xi=0.2$ and a standard deviation of $\sigma_{\xi}=0.02,0.05,0.10$ and 0.20 are shown in figure 16 . These distributions were used with a variety of different combinations of progress variable mean values and distributions and a sample result for two different turbulence time scales is shown in figure 17. While there is some variation due to the change in $\xi$ distribution, the differences are sufficiently small that they can be ignored for this model.

What has been shown is that a simple modification (shown in equation 14) to a global reaction rate can provide some basic features of a turbulence-chemistry interaction model. For a given global reaction, the two constants $B_{t}$ and $b_{t}$ may have to be calibrated, but the values given here should be a reasonable start for most forms of reactions. In addition, due to the simple form of equation 14 the additional computational cost caused by this model should be negligable. 


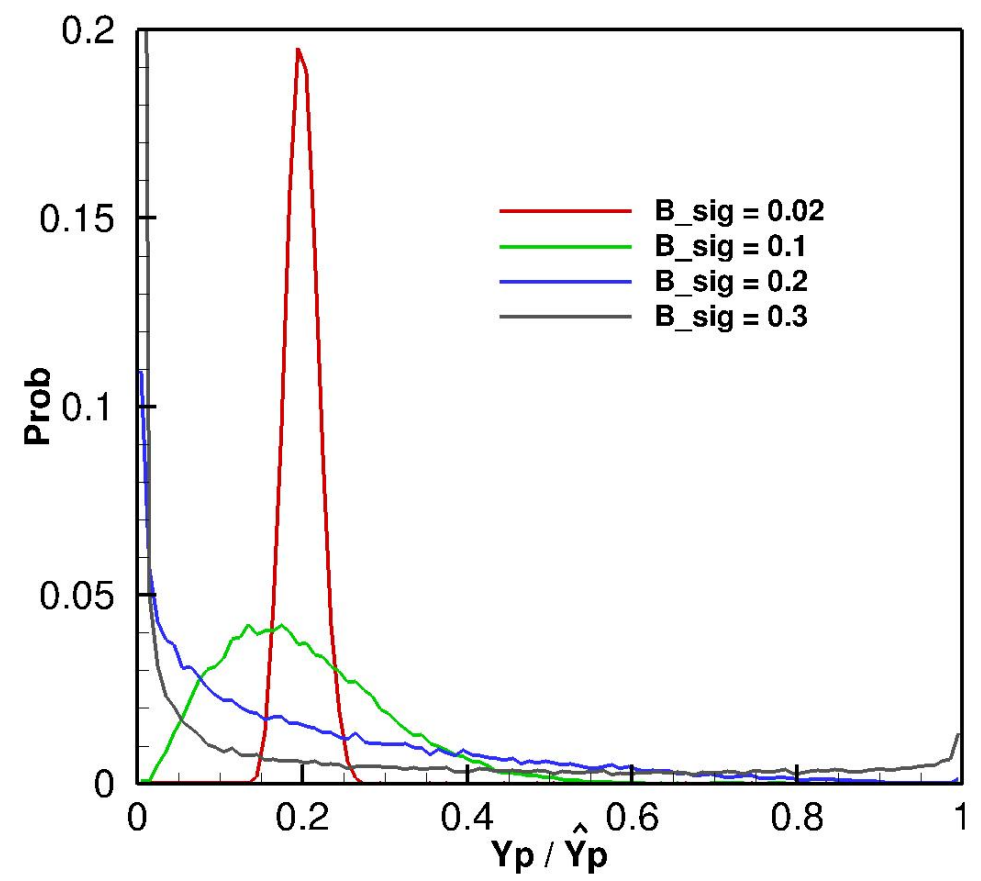

Figure 13. Selection of the distributions of progress variables used in PaSR simulations with mean of 0.2 . B_sig refers to the standard deviation of the distribution.

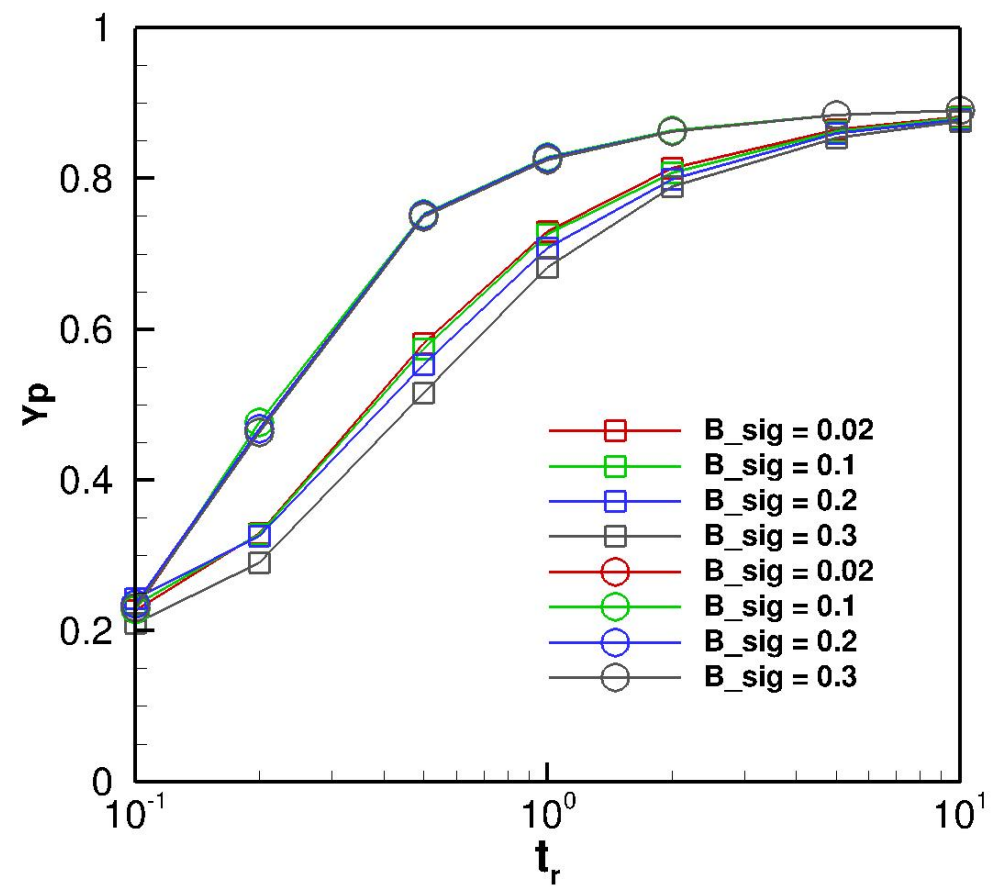

Figure 14. Variation of PaSR results due to change of inflow progress variable distributions. Square symbols are for $t_{t}=1$ and circles are for $t_{t}=10$. B_sig refers to the standard deviation of the distribution. 


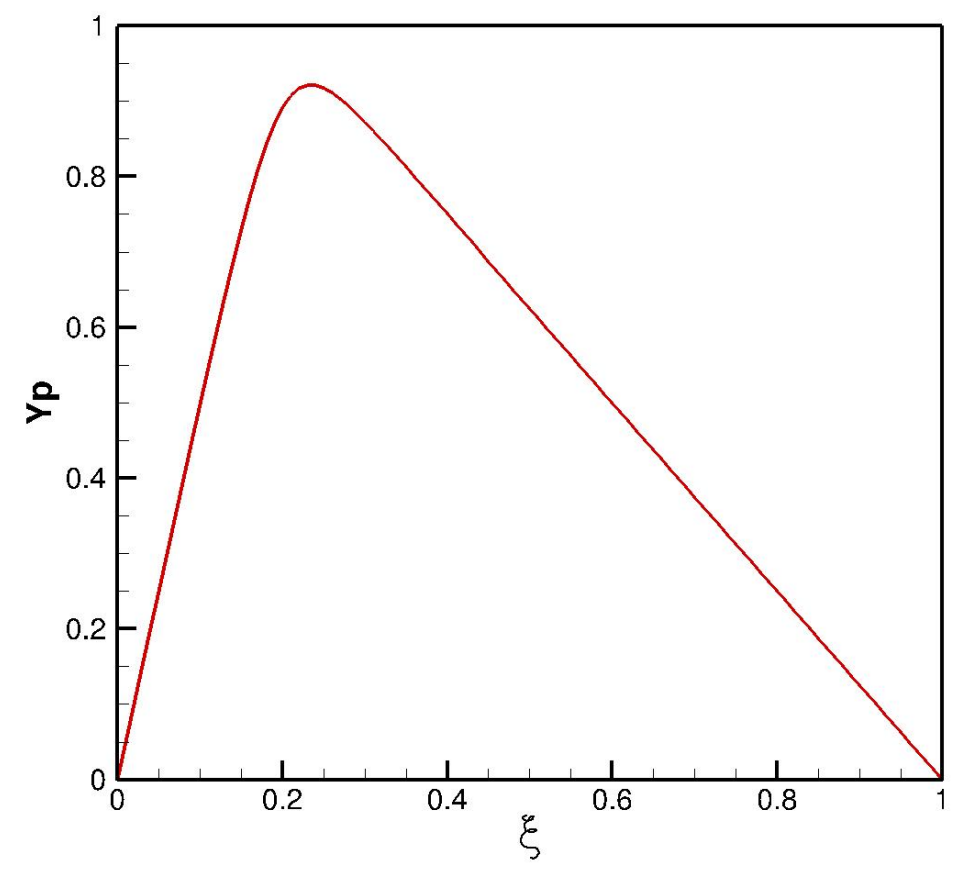

Figure 15. Maximum progress variable plotted against mixture fraction.

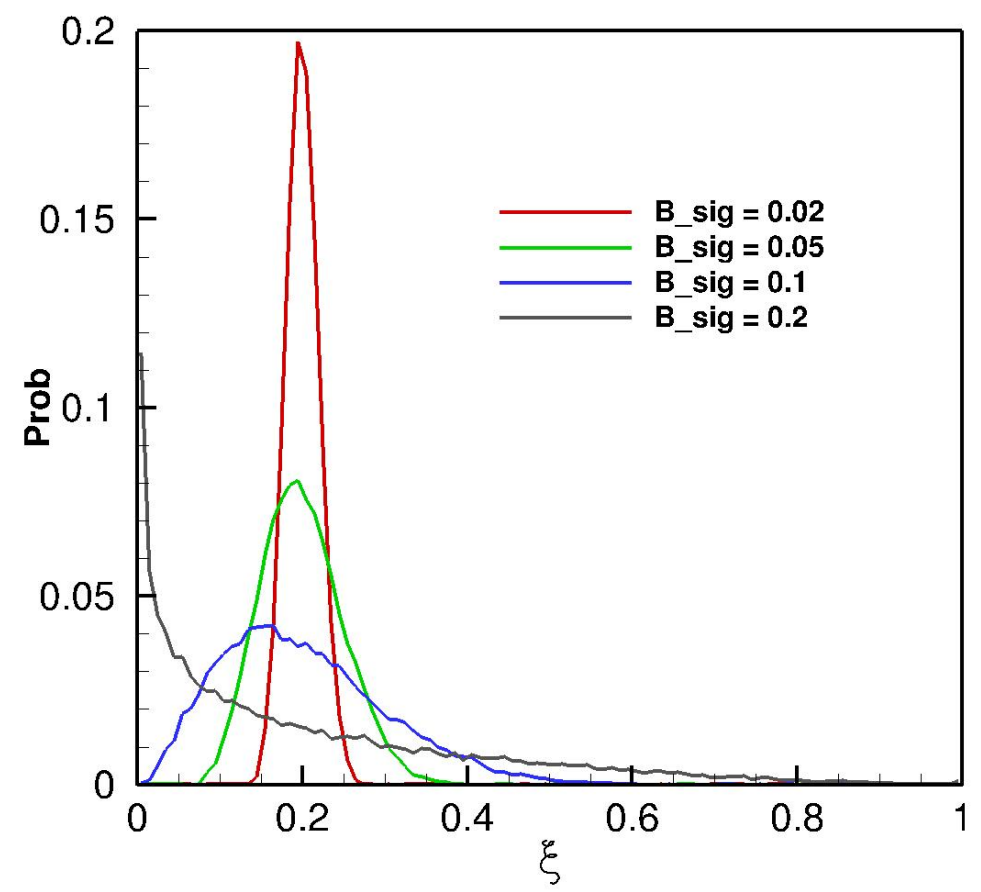

Figure 16. Distributions of mixture fraction use in PaSR distribution. B_sig refers to the standard deviation of the distribution. 


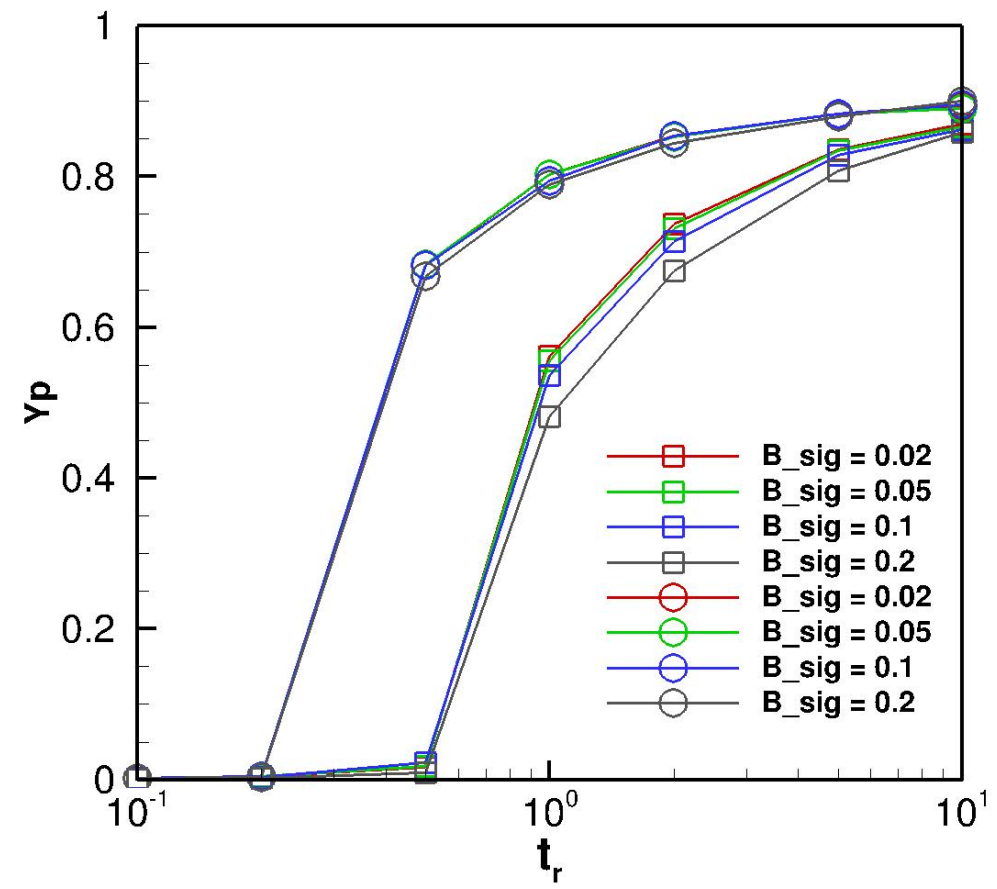

Figure 17. Variation of PaSR results due to change of inflow mixture fraction distributions. Square symbols are for $t_{t}=1$ and circles are for $t_{t}=10$. B_sig refers to the standard deviation of the distribution.

\section{Conclusions}

By comparing the mean scalar values obtained from a Perfectly Stirred Reactor (PSR) with those of a Partially Stirred Reactor (PaSR) a simple algebraic expression has been obtained that allows some of the features of the interaction between chemistry and turbulence to be modeled.

First a particle-based stochastic method was used to simulate both the PSR and PaSR. Tests were then performed to ensure the numerical accuracy of the scheme. While the results were relatively insensitive to the total number of particles used to represent the reactor, the number of particles flowing into the reactor per time step had to be limited to a small percentage of the total number to ensure accuracy. A parametric study of the physical parameters governing the performance of the reactors was then performed. It was found that the PaSR results were most sensitive to the variation of the mean inflow composition and the turbulent time scale, while the effect of varying the distribution of inflow quantities was small. As a result, a simple model has been proposed that models the effect of turbulence and mean inflow composition for a reacting flow.

Implementation of the model requires only the modification of the reaction rate term and the evaluation of a reaction rate time scale, defined as the inverse of the peak reaction rate. The model requires two constants, that may need to be tuned for different reaction rate expressions, however these values can be set via a PaSR simulation and should be constant for all uses of that kinetic mechanism. It should be noted that this expression is intended for use only with simple one-step reaction schemes and not with complex multi-step mechanisms. 


\section{References}

${ }^{1}$ Norris, A. T. and Hsu, A. T., "Comparison of PDF and Moment Closure Methods in the Modeling of Turbulent Reacting Flows," 30th AIAA/ASME/SAE/ASEE Joint Propulsion Conference, Indianapolis, Indiana, 1994, pp. AIAA-94-3356.

${ }^{2}$ Magnussen, B. F. and Hjertager, B. H., "On Mathematical Modeling of Turbulent Combustion With Special Emphasis on Soot Formation and Combustion," Sixteenth Symposium (International) on Combustion, 1976, pp. 719-729.

${ }^{3}$ Fox, R. O., Computational Models for Turbulent Reacting Flows, Cambridge University Press, Cambridge, 2003.

${ }^{4}$ Janicka, J., Kolbe, W., and Kollmann, W., "Closure of the Transport Equation for the Probability Density Function of Turbulent Scalar Fields," J. Non-Equilib. Thermodyn., Vol. 4, 1979, pp. 47-66.

${ }^{5}$ Pope, S. B., "PDF Methods for Turbulent Reactive Flows," Prog. Energy Combust. Sci., Vol. 11, 1985, pp. $119-192$.

${ }^{6}$ Kerstein, A. R., "A linear eddy model of turbulent scalar transport and mixing," Combust. Sci. Tech., Vol. 60, 1988, pp. 391-421.

${ }^{7}$ Westbrook, C. K. and Dryer, F. L., "Chemical Kinetic Modeling of Hydrocarbon Combustion," Prog. Energy Combust. Sci., Vol. 10, 1984, pp. 1-57.

${ }^{8}$ Dopazo, C., "PDF Approach for a Turbulent Axisymmetric Heated Jet: Centerline Evolution," Phys. Fluids., Vol. 18(4), 1975, pp. 397-404.

${ }^{9}$ Janicka, J., Kolbe, W., and Kollmann, W., "The Solution of a PDF-Transport Equation for Turbulent Diffusion Flames," Proceedings from the 1978 Heat Transfer and Fluid Mechanics Institute, 1978, pp. 296-312.

${ }^{10}$ Curl, R. L., "Dispersed Phase Mixing: 1. Theory and Effects in Simple Reactors," A. I. Ch. E. J., Vol. 9, 1963, pp. 175181. 